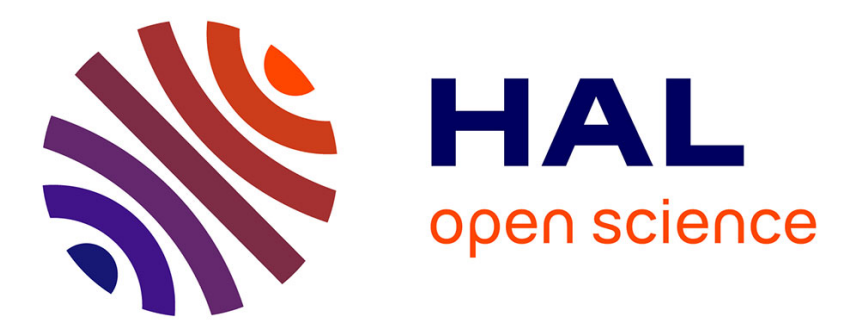

\title{
Statistical analysis of correlations and intermittency of a turbulent rotating column in a magnetoplasma device
}

Simone Magni, H. E. Roman, C. Riccardi, Th. Pierre, D. Guyomarc'H, P. Devynck

\section{- To cite this version:}

Simone Magni, H. E. Roman, C. Riccardi, Th. Pierre, D. Guyomarc'H, et al.. Statistical analysis of correlations and intermittency of a turbulent rotating column in a magnetoplasma device. 2004. hal-00001852

\section{HAL Id: hal-00001852 \\ https://hal.science/hal-00001852}

Preprint submitted on 26 Oct 2004

HAL is a multi-disciplinary open access archive for the deposit and dissemination of scientific research documents, whether they are published or not. The documents may come from teaching and research institutions in France or abroad, or from public or private research centers.
L'archive ouverte pluridisciplinaire HAL, est destinée au dépôt et à la diffusion de documents scientifiques de niveau recherche, publiés ou non, émanant des établissements d'enseignement et de recherche français ou étrangers, des laboratoires publics ou privés. 


\title{
Statistical Analysis of Correlations and Intermittency of a Turbulent Rotating Column in a Magnetoplasma Device
}

\author{
S. Magni, ${ }^{1}$ H. E. Roman, ${ }^{1}$ C. Riccardi,${ }^{1}$ Th. Pierre,${ }^{2}$ D. Guyomarc'h,${ }^{2}$ and P. Devynck ${ }^{3}$ \\ ${ }^{1}$ Dipartimento di Fisica, Universitá di Milano-Bicocca, \\ Piazza della Scienza 3, 20126 Milano, Italy \\ ${ }^{2}$ Laboratoire PIIM, CNRS et Université de Provence, 13397 Marseille, France \\ ${ }^{3}$ Association Euratom-CEA, DRFC, CEN de Cadarache, \\ 13108 St Paul Lez Durance, France
}

(Dated: October 24, 2004)

\begin{abstract}
A detailed statistical analysis of density fluctuations in the cylindrical non-fusion device 'Mistral' is performed. The experimental set-up is implemented in order to reach turbulent behavior in the rotating plasma column. Two different turbulent regimes are obtained corresponding to two selected sets of values for the anode potential and the biasing of the collecting plate. The first regime displays a slowly-rotating column characterized by the presence of a shear layer separating the plasma bulk from the scrape-off layer (SOL), the latter showing a strong intermittent behavior. The second regime corresponds to a weakly-rotating column in which coherence is lost in the plasma bulk and a standard diffusive process takes place in the SOL. These findings are supported by the calculation of the Hurst exponent of the turbulent signals using Wavelet-analysis techniques.
\end{abstract}

PACS numbers: $52.35 \mathrm{Ra}, 52.25 \mathrm{Gj}$ 


\section{INTRODUCTION}

The study of turbulence in nonfusion plasma devices is an important task to assess the understanding of transport in more complex, large-scale plasma configurations. To this end, a detailed analysis of electronic density fluctuations in the cylindrical non-fusion device Mistral is performed. The experimental set-up is implemented in order to obtain a turbulent regime in a rotating plasma column.

In this work we consider two distinct sets of discharge parameters, corresponding to the anode potential and the biasing of the collecting plate. The latter sets yield two different turbulent regimes, one corresponding to a slowly-rotating column (regime A) and a second one in which the column is only weakly rotating (regime B). In regime A, there exists a velocity shear layer sharply separating the bulk plasma from the SOL region. The former rotates nearly as a rigid body, while in the SOL a strong intermittency occurs. In regime $\mathrm{B}$, the plasma bulk loses almost all of its coherence and displays intermittency, while in the SOL density fluctuations show standard diffusive behavior.

The paper is organized as follows. In Section II the experimental set up is briefly discussed. Sect. III is devoted to a description of the statistical tools employed and Sect. IV to the discussion of the results. These include the moments of the fluctuating signals, such as mean value, standard deviation, skewness and flatness, as a function of the radial distance $r$ from the center of the cylindrical device. The calculation of Hurst exponents and the characterization of the intermittency of the corresponding time-series is presented. Finally, Sect. V contains the concluding remarks.

\section{EXPERIMENTAL SET-UP}

In Mistral, the plasma is produced by a large multipolar source, operating at a low pressure $\left(1 \div 5 \times 10^{-4}\right.$ mbar $)$ Argon discharge in the main chamber sustained by hot filament emission (see e.g. [1] for more details).

A high transparency floating grid is inserted between the source chamber and the magnetized plasma column. Primary electrons $(40 \mathrm{eV})$ overcome the floating potential of the injection grid and ionize the gas inside the column. A negative charge surplus is created close to the axis of the latter, while the ions are poorly magnetized and contribute to a 
radial loss of charges outside the column. A radial equilibrium electric field $\mathrm{E}$ then sets in. Electrons and ions drift in the same direction at the same $\mathbf{E} \times \mathbf{B}$ velocity, contributing to the rotation of the column.

In these experiments, the plasma has been confined to the linear part of the chamber (of length $1.2 \mathrm{~m}$ ) by a circular collector. The plasma diameter is limited to $14 \mathrm{~cm}$ by a metallic diaphragm (limiter), placed at the entrance of the linear part. In this way, diffusion can be investigated in the shadow of the limiter where the ionization process is no longer present. With the grid kept floating, the control parameters are the potential of the anode $V_{\mathrm{A}}$ and the biasing of the collecting plate $V_{\text {plate }}$. The corresponding values are:

$$
\begin{aligned}
& \text { Regime A : } V_{\mathrm{A}}=-8 \mathrm{~V} \text { and } V_{\text {plate }}=18 \mathrm{~V}, \\
& \text { Regime B : } \quad V_{\mathrm{A}}=-3 \mathrm{~V} \text { and } V_{\text {plate }}=10 \mathrm{~V} .
\end{aligned}
$$

The electronic saturation density is measured through a Langmuir probe, using a single pin with a polarization of $18 \mathrm{~V}$. An automated acquisition system is adopted, where the stepper-motor of the movable probe and the oscilloscope are managed by a Lab Viewßprogram.

We study stationary time records of length $N=5 \times 10^{4}$, representing electron density fluctuations at position $r$, taken at equidistant time intervals $\Delta t$. Both $\mathrm{AC}$ and $\mathrm{DC}$ signals have been considered. For regime $\mathrm{A}$, we have taken $\Delta t=2 \mu \mathrm{s}$ and obtained 20 series per radial position for DC signals, and 100 series per radial position for AC signals. For regime $\mathrm{B}$, we have taken $\Delta t=4 \mu$ s and obtained 50 series per radial position for both $\mathrm{DC}$ and $\mathrm{AC}$ signals.

\section{STATISTICAL TOOLS}

We study electron density fluctuations $\tilde{Z}(r, t)=n_{\mathrm{e}}(r, t)-\left\langle n_{\mathrm{e}}(r)\right\rangle$, where $\left\langle n_{\mathrm{e}}(r)\right\rangle$ is the mean value at $r$, and calculate few higher moments of $\tilde{Z}(r, t)$, such as the standard deviation, $\sigma_{Z}=\left\langle Z^{2}\right\rangle^{1 / 2}$, which measures the amplitude of fluctuations, the skewness, $S=\left\langle Z^{3}\right\rangle / \sigma_{Z}^{3}$, which measures the asymmetry of the signal, and the flatness, $F=\left\langle Z^{4}\right\rangle / \sigma_{Z}^{4}$, which roughly tells us about the presence of extremal events (so-called fat tailed probability distributions). The kurtosis is related to the flatness by $K=F-3$. For comparison, the Gaussian (or normal) distribution has $S=0$ and $F=3$ (i.e. $K=0$ ). 
The existence of possible long-time correlations in the turbulent signals can be searched through the determination of the Hurst $(\mathrm{H})$ exponent of the corresponding time series (see Ref. [2] as a general review). Recently, Wavelets analysis to compute $H$ has been applied to fluctuating signals taken from a simple magnetized torus and related to diffusivity [3]. In the following, we discuss the so-called wavelet fluctuation analysis [3], and disregard the variable $r$ for simplicity of notation.

In this wavelet analysis, one regards the fluctuating signal as steps performed by a random walker along a one dimensional path, where its position after $t$ time steps is obtained as

$$
W(t)=\frac{1}{\Delta t} \int_{0}^{t} d t^{\prime} \tilde{Z}\left(t^{\prime}\right) .
$$

The quantity $W(t)$ plays the role of the profile associated to a fractional Brownian motion $(\mathrm{fBm})$, which is a general model for long-range correlated walks [4]. For a typical example of a fluctuating profile see Ref. [3].

Then, we average $W(t)$ over non-overlapping time intervals of length $\ell=\tau / \Delta t$, where $\tau \geq \Delta t$ is the time-grain scale at which fluctuations are studied, yielding,

$$
B_{m}(\ell)=\frac{1}{\ell} \sum_{j=1}^{\ell} W\left(t_{(m-1) \ell+j}\right), \quad \ell \geq 1,
$$

with $1 \leq m \leq \operatorname{int}(N / \ell)$, from which we evaluate the fluctuations of such coarse-grained walks, denoted as first order wavelets, according to

$$
F_{1}^{2}(\ell)=\left\langle\left(B_{m+1}(\ell)-B_{m}(\ell)\right)^{2}\right\rangle
$$

where $\langle\ldots\rangle$ denotes the average taken over all consecutive intervals $(m, m+1)$. The exponent $H$ can be estimated from the expected scaling relation, $F_{1}(\ell) \propto \ell^{H}$. To disentangle intrinsic fluctuations from the presence of possible trends in the signal, one can also study higher order wavelets, such as

$$
\begin{aligned}
& F_{2}^{2}(\ell)=\left\langle\left[B_{m+2}(\ell)-2 B_{m+1}(\ell)+B_{m}(\ell)\right]^{2}\right\rangle \\
& F_{3}^{2}(\ell)=\left\langle\left[B_{m+3}(\ell)-3 B_{m+2}(\ell)+3 B_{m+1}(\ell)-B_{m}(\ell)\right]^{2}\right\rangle
\end{aligned}
$$

where the averages $\langle\ldots\rangle$ are performed on consecutive triplets $(m, m+1, m+2)$ and quadruplets $(m, m+1, m+2, m+3)$, respectively.

The Hurst exponent carries important information about the auto-correlations of the signal $\tilde{Z}(t)$. If $H>0.5$ the signal shows persistence, i.e. positive correlations. Values of 
$H<0.5$ indicate antipersistence, i.e. negative correlations in the data. For $H=0.5$ the signal is not correlated as, for example, for a white noise signal.

Other methods have been considered in the plasma literature to calculate $\mathrm{H}$, with different degree of accuracy and success (see e.g. [2]). The wavelet analysis has been designed to detect 'truly' long-time correlations in the signal by eliminating the effects of possible trends, and should be therefore more accurate than other methods. In addition to $H$, the intermittency in the same time-series can be investigated by means of recent-reviewed statistical tools [5].

\section{RESULTS}

Regimes A and B seem to display two different phenomenologies. These can be seem in Fig. 1 in which the mean values of density, relative fluctuations, skewness and flatness are shown as a function of radial position $r$. In the figures, also the position of the diaphragm (limiter) is indicated.

The behavior of the plasma in regime A (see Fig. 1) resembles the one described in Ref. [1]. Schematically, we can distinguish four different zones:

- Zone $1(0 \leq r \leq 6 \mathrm{~cm})$ : The plasma core, a coherent low fluctuations state.

- Zone $2(6 \leq r \leq 9 \mathrm{~cm})$ : The region very close to the column ('near' $\mathrm{SOL})$.

- Zone $3(9 \leq r \leq 11 \mathrm{~cm})$ : A high intermittent, very narrow region (within the $\mathrm{SOL}$ ).

- Zone $4(r>11 \mathrm{~cm})$ : The 'far' SOL region.

In zones 1, 2 and 4, fluctuations display a nearly-Gaussian character, while in zone 3 they show a qualitatively different shape. In the latter, fluctuations are dominated by extremal events in which a large fraction of the density, typically visualized as an arm of a spiral [1], can sometimes detache giving rise to large density blobs. These fluctuations seem to modify the diffusion behavior in the nearby zone.

In regime $\mathrm{B}$, we distinguish essentially two regimes:

- Zone $1(0 \leq r \leq 7 \mathrm{~cm})$ : The plasma core where now intermittency is present.

- Zone $2(r>7 \mathrm{~cm})$ : The shadow region in which the plasma displays normal diffusive behavior. 

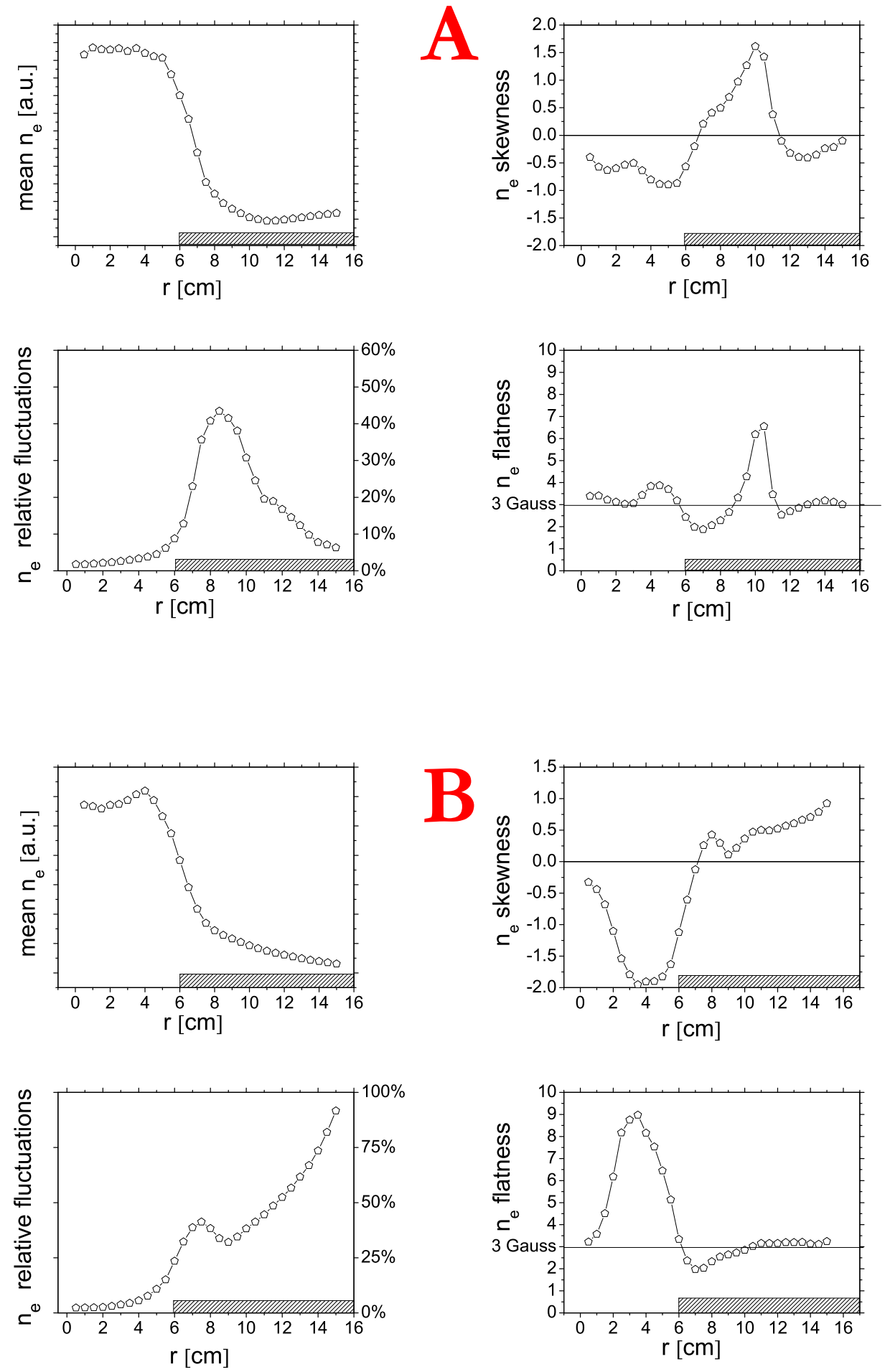

FIG. 1: Different moments of density fluctuations as a function of distance $r$. Mean value $\left\langle n_{\mathrm{e}}(r)\right\rangle$ (top left), relative fluctuations $\sigma_{\mathrm{e}} /\left\langle n_{\mathrm{e}}(r)\right\rangle$ (bottom left), skewness (top right), flatness (bottom right). The position of the limiter $(6 \leq r \leq 16 \mathrm{~cm})$ is indicated by the shadowed bar. Regime $\mathrm{A}$ (top panel), regime B (lower panel). 

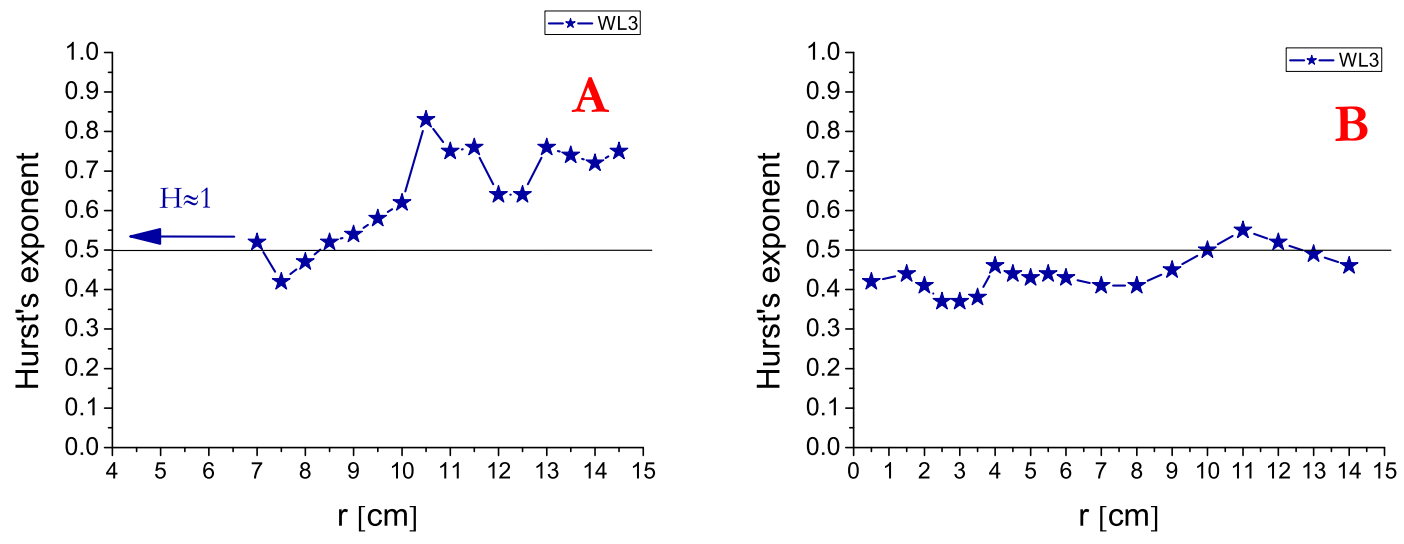

FIG. 2: Hurst exponents for regime $\mathrm{A}$ and $\mathrm{B}$, as a function of radial distance $r$, obtained using third order wavelet analysis, $F_{3}(\ell)$, Eq. (5).

Roughly speaking, regime A resembles a Tokamak configuration (where only the SOL is strongly turbulent), while regime B seems closer to an SMT (where a strong intermittency is present in the inner part of the vacuum chamber and it is strictly correlated to plasma production).

The Hurst exponents have been estimated using wavelet analysis up to third-order. The results, corresponding to third-order wavelets, Eq. (5), are reported in Fig. 2. For regime A, fluctuations display $H \approx 1$ (ballistic diffusion) in zone 1 , a consequence of the fact that the plasma rotates as a quasi-rigid body. In zone 2 , we find $H=(0.45-0.55)$ corresponding to standard diffusion. In zone $3, H=(0.75-0.83)$ pointing to a super-diffusive situation consistent with the pictures of expulsed blobs of density. Finally, in zone $4, H=(0.65-0.75)$ corresponding to a weaker super-diffusive behavior. This can be interpreted as a residual diffusive process which keeps memory of turbulent convection from the above mentioned detached structures.

For regime $\mathrm{B}$, we have a slightly anti-persistent signal with $H=(0.38-0.45)$ (subdiffusion) within the hole of the limiter $(r<7 \mathrm{~cm})$ and $H \approx 0.5$ (standard diffusion) in its shadow $(r>7 \mathrm{~cm})$ (Fig. 2).

It is interesting to study the probability distribution function (PDF) for the position $r=10.5 \mathrm{~cm}$ in the case of regime $\mathrm{A}$, which displays a fat-tail for positive fluctuations. In the following, we concentrate us on the positive side of the PDF, which is displayed 


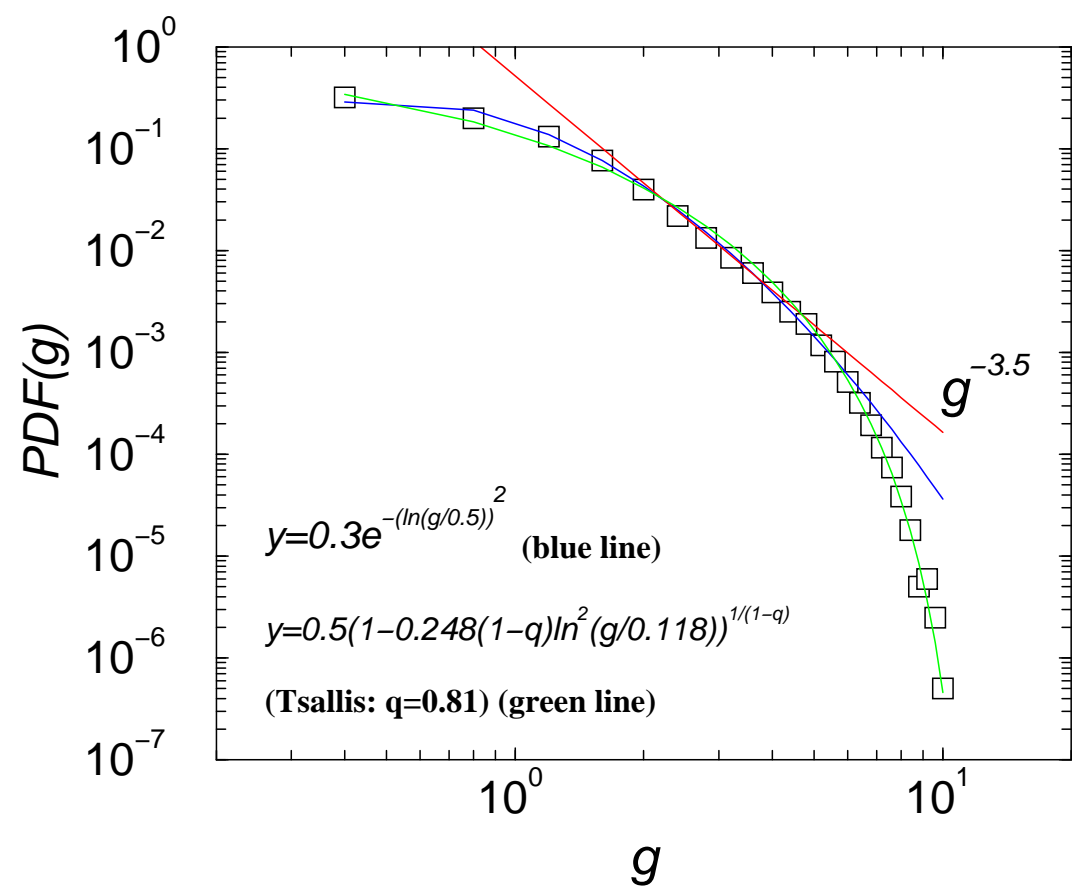

FIG. 3: The probability distribution function (PDF) for density fluctuations versus scaled variable $g=\Delta n_{\mathrm{e}} / \sigma_{\mathrm{e}}>0$, for regime A at a distance $r=10.5 \mathrm{~cm}$ (open squares). The lines at different fits to the data: power-law (red line), log-normal (blue line), and a modified log-normal (green line). The expressions used are reported in the figure.

in Fig. 3. This particular shape of the PDF has been fitted, for illustration, using three different functional forms: a power-law, a standard log-normal and a modified log-normal. The latter is assumed to be of the form of a Tsallis distribution (see e.g. Ref.[6]). It is apparent from Fig. 3 that a power-law is not consistent with the plasma data, except for a very narrow region of the scaled variable $g$. A log-normal shape approximates the plasma PDF better but fails at the extremal part of the tail. The most satisfactory fit is obtained using a modified log-normal. This behavior remains to be understood.

We have considered the question of identifying the shape of bursts in the signals (cfr. Ref. [5]). To do this, a burst is defined as a fluctuation which overcomes the threshold $3 \sigma_{\mathrm{e}}$, and perform an average over all such events within the time series corresponding to a particular position $r$. Results for the burst shapes obtained for position $r=10.5 \mathrm{~cm}$ for regime A, and for $r=3.5 \mathrm{~cm}$ for regime B, are shown in Fig. 4. Note the different widths of the burst shapes. 

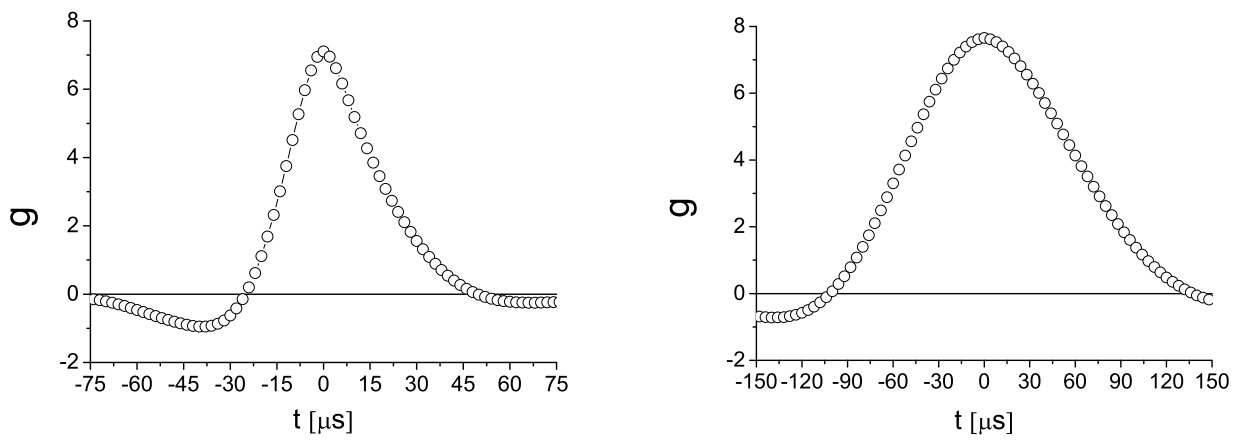

FIG. 4: The average shape of bursts of scaled density fluctuations $g$ versus time lag $t[\mu \mathrm{s}]$, at position $r=10.5 \mathrm{~cm}$ for regime A (left panel), and position $r=3.5 \mathrm{~cm}$ for regime B (right panel). A burst is considered as such when the fluctuation overcomes $3 \sigma_{\mathrm{e}}$, i.e. $g>3$, and the signal is averaged over all events present in the time series.
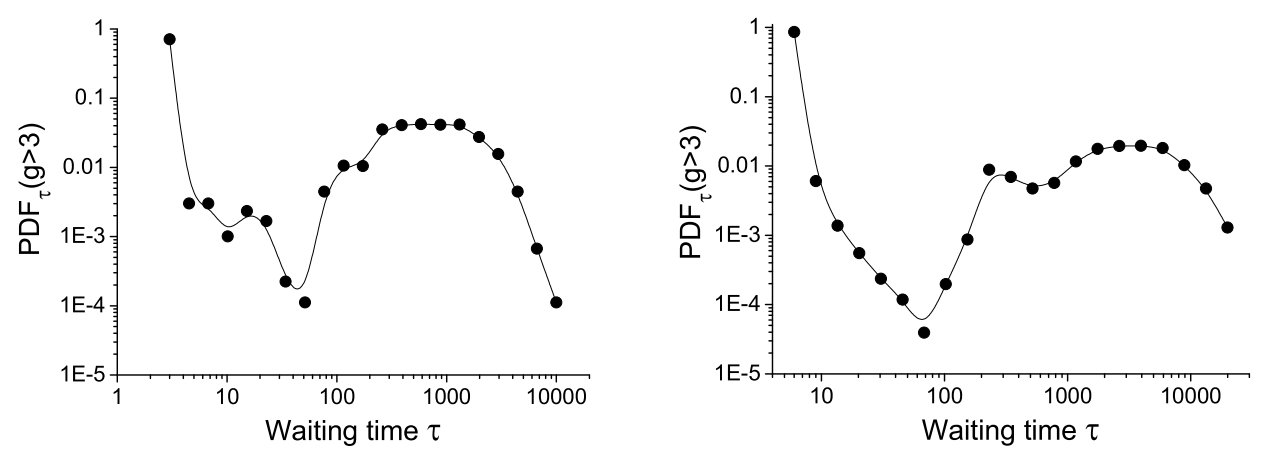

FIG. 5: The probability distribution function of waiting times between successive bursts versus $\tau$ $[\mu \mathrm{s}]$, at position $r=10.5 \mathrm{~cm}$ for regime A (left panel), and position $r=3.5 \mathrm{~cm}$ for regime B (right panel).

The waiting time $\tau$ elapsed between two successive bursts has been determined for the same positions considered in Fig. 4. The corresponding PDF are plotted in Fig. 5. Both distributions show a dip around $\tau \approx 100 \mu \mathrm{s}$, followed by a broad bump at higher time scales. To be noted is that the 'plateau' observed in the PDF at larger time scales, $\tau>100 \mu \mathrm{s}$, correspond to the time intervals where the Hurst exponents have been determined. 


\section{CONCLUSIONS}

The rotation of the plasma column seems to induce a super-diffusive process of density fluctuations in the SOL. Slowing-down the rotation, diffusion becomes closer to standard

behavior. A modified log-normal distribution seems to fit appropriately the fat-tailed PDF. The average shape of bursts, i.e. events larger than $3 \sigma$, displays a width of about $40 \mu \mathrm{s}$ for the case of strong intermittency (regime A), and of about $130 \mu$ s for the case of weaker turbulence (regime B).

[1] Th. Pierre, A. Escarguel, D. Guymarc'h, R. Barni, C. Riccardi, Phys. Rev. Lett. 92, 065004 (2004)

[2] B. A. Carreras, et al., Phys. Plasmas 6, 1885 (1999).

[3] S. Magni, C. Riccardi, H. E. Roman, Phys. Plasmas 6, 1885 (2004)

[4] J. Feder, Fractals (Plenum, New York, 1988)

[5] G. Y. Antar, et al., Phys. Plasmas 10, 419 (2003)

[6] T. Arimitsu, N. Arimitsu, Physica A 305, 218 (2002) 